initiated with the Atomic Energy Research Establishment, Harwell, who can produce ${ }^{131} \mathrm{I}$ free of ${ }^{124} \mathrm{I}$, to tackle the production of ${ }^{123}$ I-iociocholesterol and the more favoured iodocholesterol methyl derivative. ${ }^{28-29}$ Implementation of this suggestion would bring adrenal imaging into the realm of a routine screening test.

Proof of cure of primary aldosteronism can be assessed only after surgical correction. The two patients described have been followed up for a year and both have remained normotensive with a normal electrolyte pattern.

\author{
References \\ ${ }^{1}$ Conn, J W, Fournal of Laboratory and Clinical Medicine, 1955, 45, 661. \\ 2 Edmonds, C J, and Godfrey, R C, Gut, 1970, 11, 330. \\ ${ }^{3}$ Efstratopoulos, A D, Peart, W S, and Wilson, G A, Clinical Science and \\ Molecular Medicine, 1974, 46, 489. \\ ${ }^{4}$ Britton, K E, paper presented at British Nuclear Medicine Society Annual \\ Meeting, 1974. \\ ${ }^{5}$ Britton, K E, in Proceedings of Nuclear Medicine Symposium, ed K H \\ Ephraim, and H Y Oei, p 107. Utrecht, State University Publications, \\ 1975. \\ ${ }^{6}$ Britton, K E, et al, Proceedings of the 13th International Meeting of the \\ Society of Nuclear Medicine, ed T Munkner, p 29.1. Copenhagen, Fadls \\ Forlag, 1975 . \\ 'Sutton, D, British fournal of Radiology, 1975, 48, 237.
}

${ }^{8}$ Mayes, D, et al, fournal of Clinical Endocrinology and Metabolism, 1970, 30, 682 .

${ }^{9}$ Boyd, G W, et al, Lancet, 1969, 1, 213.

10 Bayliss, R I S, Edwards, O M, and Starer, F, British fournal of Radiology, 1970, 43, 531.

11 Bookstein, J J, Conn, J, and Reuter, S R, Radiology, 1968, 90, 778.

1.2 Fukuchi, S, et al, Clinical Science and Molecular Medicine, 1975, 49, 187.

${ }^{13}$ Conn, J W, in Hypertension: Mechanisms and Management, ed G Onesti, E K Kwan, and J M Moyer, p 471. New York, Grune and Stratton, $\mathbb{Q}$ 1973

11 Aitchison, J, et al, American Heart fournal, 1971, 82, 660.

15 Edmonds, C J, and Richards, P, Lancet, 1970, 2, 624

16 Beevers, D G, et al, Gut, 1975, 16, 36.

17 Beierwaltes, W H, et al, fournal of Nuclear Medicine, 1969, 10, 387.

1s Conn, J W, et al, Fournal of Clinical Endocrinology and Metabolism, 1971,0

33, 713.
19 Lieberman, L M, et al, New England fournal of Medicine, 1971, 285, $1387 \frac{\overline{\frac{\omega}{D}}}{\text {. }}$

20 Jorgensen, H, Norman, N, and Sundsfijord, J A, Acta Medica Scandinavica, 1975, 197, 345.

${ }^{21}$ Petersen, H D, et al, Acta Endocrinologica, 1975, 80, 81.

22 Kirschner, A S, Ice, R D, and Beierwaltes, W H, fournal of Nuclear $\vec{\circ}$ Medicine, 1973, 14, 713 .

${ }^{23}$ Beierwaltes, W H, et al, fournal of Nuclear Medicine, 1974, 15, 246.

${ }^{24}$ Conn, J W, et al, Archives of Internal Medicine, 1972, 129, 417.

${ }_{25}$ Conn, J W, and Cohen, E L, Archives of Internal Medicine, 1973, 131, 554 O

${ }^{26}$ Schteingart, D E, et al, Archives of Internal Medicine, 1972, 130, 384.

27 Forman, B H, et al, fournal of Nuclear Medicine, 1974, 15, 332.

${ }^{28}$ Kojima, M, et al, fournal of Nuclear Medicine, 1975, 16, 666.

29 Sarkar, S D, et al, Fournal of Nuclear Medicine, 1975, 16, 1038.

\title{
Mecillinam: a new antibiotic for enteric fever
}

\author{
P D CLARKE，A M GEDDES，D MCGHIE，J C WALL
}

British Medical fournal, 1976, 2, 14-15

\section{Summary}

Mecillinam is a new antibiotic related to the penicillins but more active than ampicillin against salmonellae, including Salmonella typhi. Mecillinam must be administered parenterally, but the ester, pivmecillinam, is absorbed from the gut. Eight patients suffering from typhoid fever and one suffering from paratyphoid fever were treated with the antibiotic, and seven responded satisfactorily. One patient could not tolerate pivmecillinam because of vomiting but there were no other adverse reactions. Serum and bile levels of mecillinam were many times the minimum inhibitory concentrations for most salmonellae. The antibiotic is a promising addition to the agents available for treating typhoid.

\section{Introduction}

Mecillinam (FL 1060) is a recently introduced 6- $\beta$-amidinopenicillanic acid antibiotic that is closely related to the penicillins but has a different mode of action and chemical structure. ${ }^{1 \cdot 2}$ It is a substituted formamidine compound and is $6-\beta$-(hexahydro$1 \mathrm{H}$-azepin-l-yl) methyleneamino penicillanic acid. Mecillinam

\footnotetext{
Department of Communicable and Tropical Diseases, East Birmingham Hospital, Birmingham B9 5ST

P D CLARKE, BM, MRCP, surgeon lieutenant commander, RN (present address: Royal Naval Hospital, Haslar, Portsmouth)

A M GEDDES, MB, FRCPED, consultant physician

Regional Public Health Laboratory, East Birmingham Hospital, Birmingham B5 9ST

D MCGHIE, MB, DIPBACT, senior microbiologist

J C WALL, FIMLS, senior technician
}

is not absorbed from the gastrointestinal tract, but for orab treatment the pivaloloxymethyl ester, pivmecillinam, which is hydrolysed enzymatically to mecillinam after absorption, i\$ satisfactory. Mecillinam is highly active against Escherichia coli $\overrightarrow{\vec{F}}$ including strains that are resistant to ampicillin. ${ }^{3}$ It has been used successfully for treating urinary tract infection. ${ }^{+}$It is very? active against salmonellae; and a few patients suffering fromp. typhoid fever have been successfully treated with the antiobitic.

We report here the use of mecillinam in nine patients suffering from enteric fever.

\section{Patients and methods}

Nine patients, aged 7 to 53 years, who were suffering from typhoid or paratyphoid fever were selected for treatment with mecillinam. Alh were admitted to East Birmingham Hospital and the diagnosis was confirmed by culture of Salmonella typhi from the blood of eight patients and Salmonella paratyphi $A$ from the blood of the ninth Eight of the nine patients were seriously ill. It was therefore considered necessary to initiate treatment with mecillinam either by intramusculan (IM) or intravenous (IV) injection.

When a clinical response had been obtained treatment was changed to oral pivmecillinam. The ninth patient, who had previously receive typhoid-paratyphoid A and B immunisation and was not seriously ill was treated throughout with the oral preparation. The antibiotic was ${ }^{+}$ given in a dose of $400 \mathrm{mg}$ every six hours except in two children aged 8 in whom the dose was $200 \mathrm{mg}$ and one adult in whom it was $600 \mathrm{mg} \stackrel{+}{7}$ Treatment was continued for 14 days and all patients remained in? hospital for at least a further two weeks, during which time specimensD of urine and stool were collected for culture. The patients were not discharged until at least six specimens were negative for the infecting organism. Further follow-up at home was arranged by the publiof health authorities. Tests of liver and renal function, together with fulp blood counts, were performed before, during, and after mecillinan. treatment.

The minimum inhibitory concentrations (MICs) of the infecting organisms for mecillinam were measured by the agar incorporation method using Wellcotest agar. In one patient serial serum concentrations of mecillinam were measured after $600 \mathrm{mg}$ of the antibiotic had been given by IV injection and again after IM injection. 


\section{Results}

Seven of the nine patients responded satisfactorily to mecillinam, six negative stool and urine cultures being obtained before discharge from hospital. Acute symptoms were relieved within 48 hours of starting treatment and all seven patients made an uncomplicated recovery. The mean time for the temperature to return to and remain normal was five days.

Because of a temporary failure of supply the patient suffering from paratyphoid fever, who had persistent vomiting, received only three IV doses of mecillinam, and oral treatment had to be started earlier than was desirable. Vomiting continued and he could not tolerate oral pivmecillinam; treatment was therefore changed to intravenous cotrimoxazole, with satisfactory recovery. The second patient who failed to respond satisfactorily was a 36-year-old Bengali who remained feverish for 10 days after starting treatment. Blood cultures continued to grow $S$ typhi for the first six days of treatment. In view of the persistent fever mecillinam was discontinued on day 10 and chloramphenicol substituted. Within 24 hours the fever had subsided. As blood, stool, and urine cultures had been negative for the preceding four days, the persistent fever might have been due to drug fever associated with mecillinam treatment. Apart from this possible case of drug fever, no untoward effects of mecillinam treatment were observed.

A patient who had been successfully treated with mecillinam had a recurrence of fever with positive blood cultures 14 days after the end of mecillinam treatment. The relapse was treated with a second two-week course of the antibiotic, with satisfactory response.

Three negative follow-up stool cultures were collected from all seven successfully treated patients one to three months after discharge from hospital. It seems unlikely, therefore, that any will become typhoid carriers.

The sensitivity of the infecting organisms to mecillinam and ampicillin is shown in table I. Table II shows serum concentrations of mecillinam after IV and IM injection of $600 \mathrm{mg}$ of the drug.

TABLE I-Sensitivity to mecillinam and ampicillin of eight $\mathrm{S}$ typhi strains and one $\mathrm{S}$ paratyphi A strain isolated from nine patients treated with mecillinam

\begin{tabular}{r|c|c|c|c|c}
\hline MIC $(\mathrm{mg} \mathrm{l}):$ & $<0.06$ & 0.06 & $0 \cdot 125$ & 0.25 & $2 \cdot 0$ \\
\hline $\begin{array}{ll}\text { Mecillinam .. } \\
\text { Ampicillin .. }\end{array}$ & 3 & 5 & $1^{*}$ & 8 & $1^{*}$ \\
\hline
\end{tabular}

*S paratyphi $A$ strain.

\section{Discussion}

None of the available chemotherapeutic agents is ideal for treating typhoid fever. Chloramphenicol remains the drug of first choice, but relapses and persistent faecal excretion are not uncommon after treatment, while irreversible aplastic anaemia is always a risk. Cotrimoxazole is a satisfactory alternative, ${ }^{7}$ but there is no good evidence that it is better than chloramphenicol. The response to ampicillin is slower than that with chloramphenicol. ${ }^{8}$ Recent reports have sugges:ed that amoxycillin ${ }^{9}$ is as effective as chloramphenicol in typhoid, but the lack of a parenteral preparation is a disadvantage in a severe septicaemic illness that is sometimes associated with vomiting.

Studies in this hospital ${ }^{5}$ have confirmed that mecillinam is highly active against salmonellae, the MIC for $S$ typhi and $S$ paratyphi varying from 0.03 to $0.5 \mathrm{mg} / \mathrm{l}$ compared with 0.2 to $4 \mathrm{mg} / \mathrm{l}$ for ampicillin. Serum levels of mecillinam many times higher than these concentrations can be readily obtained after parenteral administration of the antibiotic (table II). Oral administration of $400 \mathrm{mg}$ of pivmecillinam results in mean peak serum concentrations of mecillinam greater than $5 \mathrm{mg} / \mathrm{l}^{10}$ Satisfactory therapeutic biliary levels of mecillinam can also be achieved after oral administration. ${ }^{11}$ We have confirmed this in a postoperative patient with a $\mathrm{T}$-tube in the common bile duct in whom a peak mecillinam bile level of $6.2 \mathrm{mg} / 1$ was obtained two hours after a $400-\mathrm{mg}$ dose of the antibiotic (unpublished data).

The present preliminary report has confirmed the efficacy and safety of mecillinam in enteric fever but does not immediately suggest that mecillinam is any better than other available therapeutic agents. The mean time of five days for the successfully treated patients to become afebrile compares favourably with the response to chloramphenicol and co-trimoxazole.' Chloramphenicol-resistant $S$ typhi strains, such as those that caused the large typhoid epidemic in Mexico, are sensitive to ampicillin ${ }^{12}$ and mecillinam will probably also be effective against these organisms. Further information is required on this matter. The patients in the present study will be followed up for several more months by the public health authorities, and it will be interesting to learn whether any become persistent excreters of the organism. Mecillinam biliary levels are high, ${ }^{11}$ and the numbers of chronic typhoid carriers might be reduced with this drug. Similarly, mecillinam, because of its safety and biliary excretion, might be extremely useful in the long-term management of typhoid carriers.

Persistently positive blood cultures for up to 10 days after the start of treatment are not uncommon in typhoid fever and have been reported with chloramphenicol ${ }^{13}$ and amoxycillin. ${ }^{9}$ In one patient in this study positive cultures persisted for six days after the start of mecillinam treatment despite high serum levels of the antibiotic and satisfactory serum antibacterial activity. This phenomenon may be a feature of the enteric fever rather than the treatment and might be due to the intermittent release into the blood stream of organisms from sites (such as the reticuloendothelial system) relatively inaccessible to chemotherapeutic agents. In studies with Escherichia coli, however, Greenwood and $\mathrm{O}^{\prime} \mathrm{Grady}^{14}$ exposed sensitive organisms to mecillinam and subsequently found phenotypic variants which were no longer inhibited by the antibiotic. The latter explanation, however, seems unlikely in this case, as the $S$ typhi remained fully sensitive to mecillinam, even when cultured on the sixth day of treatment. Furthermore, Greenwood and O'Grady's studies were entirely in vitro and their clinical significance is thus uncertain.

Leo Laboratories kindly provided the antibiotics in this study. Thanks are due to Dr J G P Hutchison and Dr R Fothergill for their co-operation. Dr Richard Wise, Dudley Road Hospital, Birmingham, carried out the mecillinam assays.

Requests for reprints should be sent to AMG.

\section{References}

${ }^{1}$ Lund, F, and Tybring, L, Nature, New Biology, 1972, 236, 135.

2 Park, J T, and Burman, L, Biochemical and Biophysical Research Communications, 1973, 51, 863.

${ }^{3}$ Greenwood, D, et al, fournal of Clinical Pathology, 1974, 27, 192.

4 Verrier Jones, E R, and Asscher, A W, Journal of Antimicrobial Chemotherapy, 1975, 1, 193.

5 McGhie, D, et al, Chemotherapy, ed J D Williams and A M Geddes. New York, Plenum Press, 1976, 5, 199.

${ }^{6}$ Limson, B M, Phillipine fournal of Internal Medicine, 1973, 11, 29.

; Geddes, A M, et al, British Medical fournal, 1971, 3, 451.

${ }^{8}$ Geddes, A M, and Murdoch, J, McC, Postgraduate Medical fournal, 1964, 40, (December suppl), p 81.

${ }^{9}$ Scragg, J N, and Rubidge, C J, American fournal of Tropical Medicine and Hygiene, 1975, 24, 860 .

${ }^{10}$ Roholt, K, Nielsen, B, and Kristensen, E, Chemotherapy, 1975, 21, 146.

11 Sales, J E L, Wilson, A, and Rimmer, D. Paper read at 9th International Congress of Chemotherapy, London, July, 1975.

${ }^{12}$ Anderson, E S, and Smith, H R, British Medical fournal, 1972, 3, 329.

13 Watson, K C, Fournal of Clinical Pathology, 1955, 8, 55.

${ }^{14}$ Greenwood, D, and O'Grady, F, fournal of Clinical Pathology, 1973, 26, 1.

TABLE II-Serum mecillinam levels after intravenous and intramuscular injection of 600 mg of mecillinam

\begin{tabular}{|c|c|c|c|c|c|c|c|c|c|c|c|}
\hline Time after dose $(\mathrm{min})$ & Before dose & 5 & 10 & 20 & 30 & 45 & 60 & 90 & 120 & 180 & 240 \\
\hline Serum level $(\mathrm{mg} / \mathrm{l}):\left\{\begin{array}{l}\mathrm{IV} \\
\mathrm{IM}\end{array}\right.$ & $\begin{array}{l}0 \\
0\end{array}$ & 46 & 44 & 30 & $\begin{array}{c}27 \\
13.5\end{array}$ & 22 & $\begin{array}{c}14 \cdot 5 \\
15\end{array}$ & $12 \cdot 2$ & $\begin{array}{l}4 \cdot 6 \\
8 \cdot 8\end{array}$ & 5 & $\begin{array}{l}3 \cdot 5 \\
3\end{array}$ \\
\hline
\end{tabular}

